\title{
Review of the Joint National Committee 8 Guideline for Hypertension
}

\author{
Rebecca McDonald MD
}

The Joint National Committee (JNC) 8 guideline released in December 2013 is considered by many to be a drastic change in the approach to the management of hypertension. The panel members appointed to the 8th Joint National Committee set out to formulate an evidence-based guideline by reviewing randomized controlled trials found in original publications. The initial search reviewed literature published between 1/1966 and 12/2009. Excluded studies included those with a small sample size < 100 , a follow-up period of less than one year, systematic reviews, and meta-analyses. In addition, two independent searches identified literature published between 12/2009 and 8/2013.

\section{Comparing JNC 7 AND JNC 8}

JNC 8 does not attempt to redefine the basic concepts of hypertension or prehypertension which were defined in JNC 7. The panel believes that the 140/90 mm Hg definition from JNC 7 remains reasonable. However, the new guidelines focus on establishing new thresholds for initiating pharmacologic treatment and new blood pressure goals for certain subpopulations. Whereas the JNC 7 specified separate treatment goals for "uncomplicated" hypertension and for subsets with various comorbid conditions, the JNC 8 gives similar treatment goals for all hypertensive populations except when the review of evidence supports different goals for a particular subpopula-

\begin{tabular}{l}
$\begin{array}{l}\text { Corresponding author: Rebecca McDonald, MD } \\
\text { Contact Information: Rebecca.mcdonald@ } \\
\text { ttuhsc.edu }\end{array}$ \\
DOI: $10.12746 /$ swrccc2014.0208.108 \\
\hline
\end{tabular}

tion. Within the general population, the JNC 8 distinguishes between different blood pressure goals for those 60 years or older and those under 60 years. In addition, specific goals of treatment are specified for patients with chronic kidney disease (CKD) and with diabetes mellitus (DM).

The recommended drug therapy in the JNC 8 guideline differs from the previous guideline. JNC 7 recommended five classes to be considered as initial therapy but recommended thiazide-type diuretics as initial therapy for most patients without a compelling indication for another class. JNC 8, on the other hand, recommends four specific medication classes to be used as initial therapy. These four classes include angiotensin converting enzyme inhibitors (ACEI) or angiotensin receptor blockers (ARBs), calcium channel blockers, and thiazide diuretics. Within JNC 8 , there are also specific medication classes recommended based on evidence review with regard to race (black versus nonblack), CKD, and DM. This is in contrast to JNC 7 which not only specified particular medication classes for those with DM and CKD but also made specific recommendations based on the presence of other compelling indications, such as heart failure, myocardial infarction, stroke, and high cardiovascular disease risk.

\section{Three Questions Used in Formulation of the GUIDELINE}

The JNC 8 makes nine recommendations. The following three questions were used by panel members to guide the formulation of eight of the nine recommendations:

1. In adults with hypertension, does initiating antihy- 
pertensive pharmacologic therapy at specific blood pressure thresholds improve health outcomes? (Recommendations 1-5)

2. In adults with hypertension, does treatment with antihypertensive pharmacologic therapy to a specified blood pressure goal lead to improved health outcomes? (Recommendations 1-5)

3. In adults with hypertension, do various antihypertensive drugs or drug classes differ in comparative benefits and harms on specific health outcomes? (Recommendations 6-8)

\section{The ReCOMmENDATIONS}

Recommendations 1 through 5 specify blood pressure thresholds for initiating pharmacologic treatment and also blood pressure goals once treatment is initiated.

Recommendation 1: For the general population aged $\geq 60$ years

-Threshold for initiating pharmacologic treatment: systolic blood pressure (SBP) of $\geq 150$ or diastolic blood pressure (DBP) of $\geq 90$.

-Treatment goal: SBP $<150$ and DBP $<90$. \{Strong recommendation- Grade $\mathrm{A}\}$

Corollary Recommendation: In the general population aged $\geq 60$ years, if the pharmacologic treatment results in lower achieved SBP such as < $140 \mathrm{~mm} \mathrm{Hg}$, and treatment is being well tolerated without adverse effects on health or quality of life, the treatment does not need to be adjusted.

\{Expert opinion- Grade E\}

Recommendation 2: For the general population aged $<60$ years

-Threshold for initiating pharmacologic treatment: DBP of $\geq 90$.
-Treatment goal: DBP $<90$.

For ages 30-59 years \{Strong recommendation- Grade A\}

E\}

For ages 18-29 years \{Expert opinion- Grade

Recommendation 3: For the general population aged $<60$ years

-Threshold for initiating pharmacologic treatment: SBP of $\geq 140$.

-Treatment goal: SBP $<140$.

\{Expert opinion- Grade E\}

Recommendation 4: For population aged $\geq 18$ years with CKD

-Threshold for initiating pharmacologic treatment: SBP of $\geq 140$ or $\mathrm{DBP} \geq \mathbf{9 0}$.

-Treatment goal: SBP $<140$ and DBP $<90$. \{Expert opinion- Grade E\}

Recommendation 5: For population aged $\geq 18$ years with DM:

-Threshold for initiating pharmacologic treatment: SBP of $\geq 140$ or $\mathrm{DBP} \geq 90$.

-Treatment goal: SBP $<140$ and DBP $<90$. \{Expert opinion- Grade E\}

Recommendations 6 through 8 specify the recommended initial pharmacologic classes to choose according to certain subpopulations.

Recommendation 6: In general nonblack population, including those with DM

-Drug classes: Thiazide-type diuretic, calcium channel blocker (CCB), ACE-inhibitor (ACEI) or ARB

$\{$ Moderate recommendation- Grade B\} 


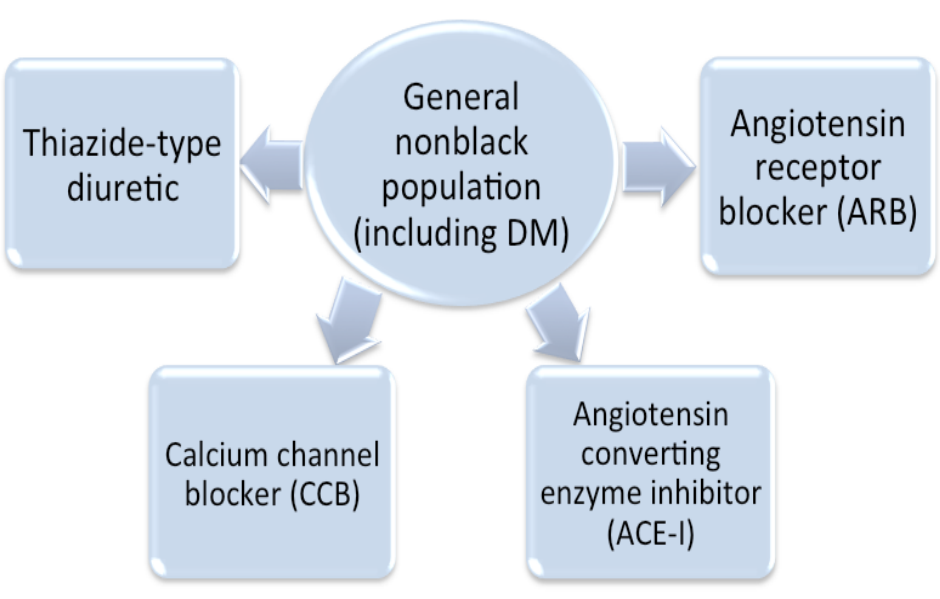

Recommendation 7: In general black population, including those with DM:

-Drug classes: Thiazide-type diuretic or CСB

\{For general black population- Moderate recommendation- Grade B\}

\{For black patients with DM- Weak recommendation- Grade C\}

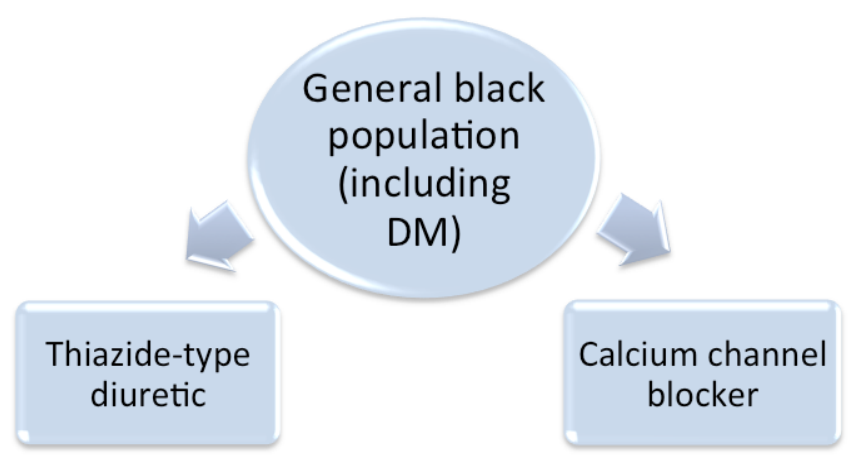

Recommendation 8: In population aged $\geq 18$ years with CKD

-Drug classes: Initial (or add-on) pharmacologic treatment should include an ACE-I or ARB to improve kidney outcomes (regardless of race or diabetes status).

\{Moderate recommendation- Grade B\}

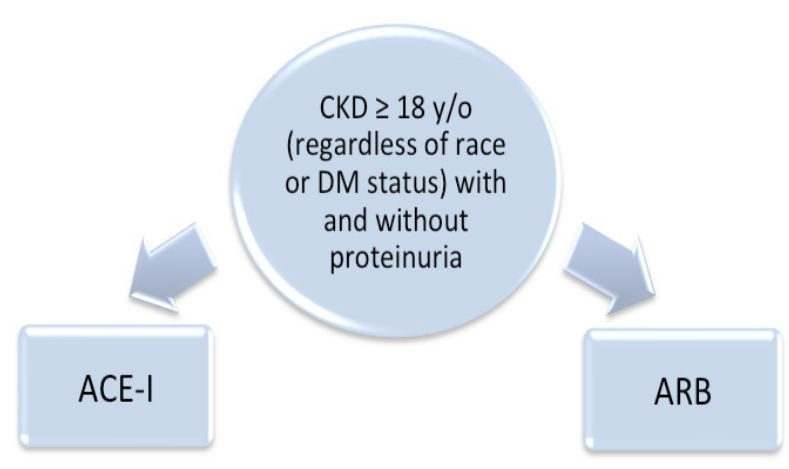




\section{Recommendation 9}

This recommendation stresses the importance of attaining and maintaining the goal blood pressure. If the goal is not attained in one month, JNC 8 recommends adjusting the medication regimen by either increasing the initial drug dose or adding a second drug that is listed in Recommendation 6. If the blood pressure goal is not reached using two drugs, then it is recommended to add a third drug from the list. This recommendation also notes that an ACE-I and ARB should not be used together in the same patient.

If the blood pressure goal is not reached using the drugs from the classes noted in Recommendation 6 due to the need for more than three drugs or due to contraindication, then JNC 8 does allow the use of other antihypertensive drugs from other classes. Referral to a hypertension specialist may be indicated if the blood pressure goal is not achieved or for more complicated patients who may also need additional clinical consultation.

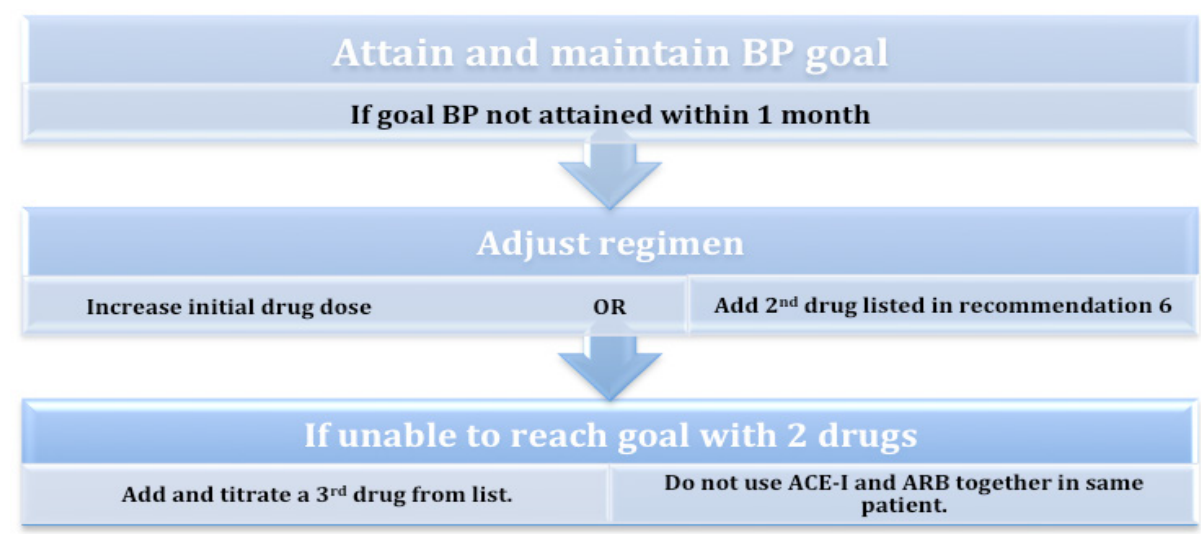

\section{Conclusion}

Hypertension is one of the most significant treatable contributors to disease and death. In addition to pharmacologic treatment, it is important to remind patients with hypertension about the key role of a healthy diet, weight control, and regular exercise for better control of blood pressure. The panel members of JNC 8 support the recommendations of the 2013 Lifestyle Work Group. In conclusion, one should keep in mind that, as with any guideline, the JNC 8 recommendations do not replace clinical judgment and that decisions regarding the management of patients should consider an individual's clinical characteristics and circumstances.
Author Affiliation: Rebecca McDonald is a general internist in the Department of Internal Medicine at TTUHSC.

Received: 08/02/2014

Accepted: 09/07/2014

Reviewers: Dolores Buscemi MD

Published electronically: 10/15/2014

Conflict of Interest Disclosures: None

\section{REFERENCE}

1. James PA, Oparil S, Carter BL, et al. 2014 Evidence-Based Guideline for the Management of High Blood Pressure in Adults: Report From the Panel Members Appointed to the Eighth Joint National Committee (JNC 8). JAMA. 2014; 311(5):507-520. doi:10.1001/jama.2013.284427. 